Volumen 25, No 1, Páginas 73-76

IDESIA (Chile) Enero - Abril 2007

\title{
TABELA DE VIDA DE FERTILIDADE DE TRICHOGRAMMA EXIGUUM (HYMENOPTERA: TRICHOGRAMMATIDAE)
}

\author{
LIFE TABLE OF THE TRICHOGRAMMA EXIGUUM \\ (HYMENOPTERA: TRICHOGRAMMATIDAE)
}

\author{
Harley Nonato de Oliveira $^{1}$; Dirceu Pratissoli ${ }^{1 *}$; Carlos Alexandre Colombi ${ }^{1}$; \\ Ricardo Antonio Polanczyk ${ }^{1}$; Leandro Pin Dalvi ${ }^{1}$
}

\begin{abstract}
RESUMO
Trichogramma é um dos mais importantes inimigos naturais e tem sido utilizado com sucesso em diversos programas de controle biológico de pragas em todo o mundo. O objetivo foi obter uma tabela de vida para Trichogramma exiguum em diferentes gerações, quando criados em ovos dos hospedeiros alternativos Anagasta kuehniella e Sitotroga cerealella. O experimento foi desenvolvido a $25 \pm 11^{\circ} \mathrm{C}, 70 \pm 10 \%$ de umidade relativa e 14 horas de fotofase, e foi avaliado a duração média de uma geração (T), taxa líquida reprodutiva (Ro), razão infinitesimal de aumento $\left(\mathrm{r}_{\mathrm{m}}\right)$ e a razão finita de aumento $(\lambda)$. Os resultados mostraram que T. exiguum foi mais eficiente quando se utilizou o hospedeiro A. kuehniella, mostrando ser esse inseto o mais indicado para a criação massal desse parasitóide.
\end{abstract}

Palavras chave: Tabela de vida, Trichogramma, controle biológico, hospedeiro.

\begin{abstract}
Trichogramma it is one the most important natural enemies and has been successfully utilized for biological control of several pests world-wide. The objective was to construct a life table of Trichogramma exiguum in different generations, when reared on eggs of Anagasta kuehniella and Sitotroga cerealella. The research was development at $25 \pm 1^{\circ} \mathrm{C}, 70 \pm 10 \%$ relative humidity and 14 hours of photophase and were evaluated the duration of a generation $(T)$, liquid reproductive rate (Ro), infinitesimal rate of increase $\left(r_{m}\right)$ and finite rate of increase $(\lambda)$. The results showed that T. exiguum were more efficient in eggs of A. kuehniella, showing to be these insect the most indicated to establish a massal rearing.
\end{abstract}

Key words: Life table, Trichogramma, biological control, host.

O gênero Trichogramma é de grande interesse, principalmente por ser utilizado no controle de pragas de importância econômica da ordem Lepidoptera, além de ser facilmente criado em laboratório (Navarro, 1998; Oliveira et al., 2000). Espécies de Trichogramma estão associadas a 42 hospedeiros no continente sul-americano, sendo Trichogramma exiguum Pinto \& Platner (Hymenoptera: Trichogrammatidae) uma das que possuem maior número, parasitando ovos de doze espécies, associadas a sete culturas (Zucchi \& Monteiro, 1997).
Para a avaliação de inimigos naturais, as tabelas de vida de fertilidade são freqüentemente usadas (Hansen et al., 1999), sendo de grande valia para a compreensão da dinâmica populacional das espécies, representando um excelente método para estudos biológicos inter e intraespecíficos (Pratissoli \& Parra, 2000). Sendo assim, este trabalho teve por objetivo obter a tabela de vida de fertilidade de T. exiguum, avaliando-se o potencial desse parasitóide em diferentes gerações, quando criado em ovos dos hospedeiros alternativos Sitotroga cerealella (Oliv.) (Lepidoptera: Gelechiidae) e Anagasta kuehniella (Zeller) (Lepidoptera: Pyralidae).

1 Laboratório de Entomologia, Departamento de Fitotecnia, Centro de Ciências Agrárias da Universidade Federal do Espírito Santo (CCA-UFES), Alto Universitário s/n, Alegre, ES. CEP 29500-000. E-mail: pratissoli@ cca.ufes.br

* Autor para correspondência.

Fecha de Recepción: 19 Abril 2006

Fecha de Aceptación: 23 Mayo 2006 
Trichogramma exiguum utilizados na realização do experimento foram coletados em ovos de $A$. kuehniella em plantios comerciais de tomate, em Piaçú, distrito de Muniz Freire, Espírito Santo. Esta espécie foi mantida no Laboratório de Entomologia do Centro de Ciências Agrárias da Universidade Federal de Espírito Santo (CCA-UFES), localizado em Alegre, ES, por 30 gerações em ovos dos hospedeiros alternativos A. kuehniella e S. cerealella, em câmaras climatizadas com temperatura de $25 \pm 1^{\circ} \mathrm{C}, 70 \pm 10 \%$ de umidade relativa e 14 horas de fotofase.

Para a realização do experimento, foram individualizadas 25 fêmeas em cada uma das gerações avaliadas em ambos os hospedeiros. Cada fêmea recém emergida foi acondicionada em um tubo de vidro $(4,0 \times 0,7 \mathrm{~cm})$ contendo mel, sendo o mesmo fechado com filme plástico de PVC. Para cada fêmea, foram oferecidos 40 ovos do hospedeiro no qual estava sendo mantida, que foram colados com goma arábica diluída a $10 \%$ em um retângulo de cartolina $(0,5 \times 3,5 \mathrm{~cm})$ azul celeste, sendo os ovos de A. kuehniella inviabilizados em lâmpada germicida por 50 minutos.

A cada 24 horas de parasitismo, essas cartelas foram substituídas por outras contendo ovos não parasitados. Esse procedimento foi repetindo até a morte de cada fêmea de T. exiguum. As cartelas já submetidas ao parasitismo, foram acondicionadas em tubos de vidro $(8,5 \times 2,4 \mathrm{~cm})$ e mantidas nas mesmas condições da criação massal do parasitóide até a emergência dos adultos.

A metodologia utilizada para obter a tabela de vida de fertilidade foi baseada na descrita por Silveira Neto et al. (1976), onde avaliou-se a duração de uma geração, o parasitismo (\%), a viabilidade (\%) e a razão sexual do mesmo, na $10^{\circ}, 20^{\circ}$ e $30^{\circ}$ geração de laboratório, quando criado em ovos dos hospedeiros alternativos A. kuehniella e S. cerealella, para calcular a taxa líquida de reprodução (Ro), a razão infinitesimal de aumento ( $\mathrm{Rm})$, razão finita de aumento $(\lambda)$ e duração média de uma geração $(\mathrm{T})$.

A duração média de uma geração (T) de $T$. exiguum, quando criado em ovos de A. kuehniella por trinta gerações variou de 11,44 a 11,92 (Tabela 1). Estes valores são próximos aos encontrados por Zhang et al. (2001) para Trichogramma clilonis Ishii, Trichogramma pretiosum Riley e Trichogramma ostriniae Pang \& Chen criados em ovos de Plutela xylostella (L.) (Lepidoptera:
Plutellidae) e por Haile et al. (2002) para Trichogramma bournieri Pintureau \& Babault e Trichogramma sp. nr. mwanzai Schulten \& Feijen quando criados em ovos de $S$. cerealella. Quando se utilizou o hospedeiro $S$. cerealella essa variação foi de 10,48 a 10,99 (Tabela 1). Estes valores são próximos aos encontrados por Zhang et al. (2001) para Trichogramma neridai Pintureau criado em ovos de Plutela xylostella (L.) (Lepidoptera: Plutellidae) e por Pratissoli et al. (2004) para $T$. pretiosum e Trichogramma acacioi Brun, Moraes \& Soares, em ovos de A. kuehniella. Estes valores indicam que o hospedeiro A. kuehniella proporciona ao parasitóide uma duração média de uma geração $(\mathrm{T})$, aproximadamente, um dia maior que o hospedeiro $S$. cerealella.

A taxa líquida reprodutiva (Ro), que é um importante parâmetro para avaliar uma criação, pois não sofre influencias abióticas, sendo uma característica inata da população, apresentou melhores resultados quando se utilizou o hospedeiro A. kuehniella, com valores de 63,$88 ; 42,23$ e 61,13 na $10^{\circ}, 20^{\circ}$ e $30^{\circ}$ geração, respectivamente (Tabela 1). Estes valores são próximos aos encontrados por Park \& Oatman (1982) para T. brevicapillum Pinto \& Platner e T. pretiosum em ovos de T. ni. No entanto, quando T. exiguum foi criado em $S$. cerealella, observou-se os menores valores para a taxa líquida reprodutiva $\left(29,03 ; 35,05\right.$ e 16,44 na $10^{\circ}, 20^{\circ}$ e $30^{\circ}$ geração, respectivamente), que são próximos aos encontrados por Pratissoli et al. (2004) para T. pretiosum, também em ovos de $S$. cerealella, e por Scholler \& Hassan (2001) para Trichogramma evanescens Westwood sobre Ephestia elutella (Hübner) (Lepidoptera: Pyralidae). Os maiores valores apresentados pelo parasitóide, quando criado em A. kuehniella, ao longo das gerações, mostra que este hospedeiro proporciona um maior potencial de produção de fêmeas, o que pode contribuir para uma maior eficiência nos programas de controle biológico, pois são as fêmeas as responsáveis pelo parasitismo dos ovos das pragas.

A razão infinitesimal de aumento $\left(\mathrm{r}_{\mathrm{m}}\right)$ variou de 0,33 a 0,36 em A. kuehniella e de 0,29 a 0,32 com o hospedeiro $S$. cerealella, para as três gerações avaliadas (Tabela 1). Pratissoli et al. (2004) relataram valores de 0,35 e 0,32 para T. acacioi e T. pretiosum, respectivamente, quando mantidos em ovos de $A$. kuehniella. Haile et al. (2002) relatam valores de 0,31 para Trichogramma bournieri Pintureau \& Babault e 0,30 para Trichogramma sp. nr. mwanzai 
Tabela 1

Duração da geração $(T)$, taxa líquida reprodutiva $\left(R_{\mathrm{o}}\right)$, razão infinitesimal de incremento $\left(\mathbf{r}_{\mathrm{m}}\right)$, e razão finita de aumento $(\lambda)$ de Trichogramma exiguum (Hymenoptera: Trichogrammatidae) em ovos de Anagasta kuehniella (Lepdopitera: Pyralidae) e Sitotroga cerealella (Lepdopitera: Gelechiidae) em diferentes gerações. $25 \pm 1{ }^{\circ} \mathrm{C}$, umidade relativa de $70 \pm 10 \%$ e fotofase de 14 horas

\begin{tabular}{|c|c|c|c|c|}
\hline Gerações & $\mathbf{T}$ (dias) & Ro & $\mathbf{r}_{\mathbf{m}}$ & $\lambda$ \\
\hline \multicolumn{5}{|c|}{ Anagasta kuehniella } \\
\hline 10 & 10,05 & 65,47 & 0,42 & 1,52 \\
\hline 20 & 9,90 & 43,59 & 0,34 & 1,41 \\
\hline 30 & 10,00 & 66,35 & 0,42 & 1,52 \\
\hline \multicolumn{5}{|c|}{ Sitotroga cerealella } \\
\hline 10 & 10,00 & 32,90 & 0,35 & 1,42 \\
\hline 20 & 10,20 & 41,00 & 0,36 & 1,44 \\
\hline 30 & 9,80 & 20,73 & 0,31 & 1,36 \\
\hline
\end{tabular}

Schulten \& Feijen quando criados em ovos de $S$. cerealella. Essas diferenças na razão infinitesimal de aumento, ao longo das gerações, são devidas ao valor do intervalo de tempo entre cada geração $(\mathrm{T})$ e valor de Ro (Birch, 1948). Estes valores demonstram que o hospedeiro A. kuehniella proporciona uma maior razão de aumento populacional que o hospedeiro $S$. cerealella, tornando o primeiro hospedeiro mais indicado para um incremento populacional e criação massal.

Os valores observados para a razão finita de aumento $(\lambda)$ do parasitóide foram de 1,$44 ; 1,39$ e 1,41 para A. kuehniella e de 1,38; 1,38 e 1,33 para S. cerealella, para a $10^{\circ}, 20^{\circ}$ e $30^{\circ}$ geração, respectivamente (Tabela 1). Estes resultados apresentam relação direta com a taxa líquida reprodutiva e com a razão infinitesimal de aumento, pois estes são os principais fatores que influenciam no valor de $\lambda$. Os valores encontrados para T. exiguum criado em ovos de A. kuehniella foi maior que o relatado por vários autores, assim como Zhang et al. (2001), para Trichogramma chilonis Ishii, T. nerudai, T. pretiosum e $T$. ostriniae criados em ovos de $P$. xylostella, por Haile et al. (2002) para T. bournieri e Trichogramma sp. nr. mwanzai em ovos de S. cerealella, próximos aos relatados por Pratissoli et al.
(2004) utilizando T. acacioi em ovos A. kuehniella e por Scholler \& Hassan (2001) para Trichogramma evanescens Westwood sobre Ephestia elutella (Hübner) (Lepidoptera: Pyralidae). Os valores observados para a razão finita de aumento de $T$. exiguum criado em $S$. cerealella, foram próximos aos relatados por Pratissoli et al. (2004) T. pretiosum em ovos A. kuehniella, por Haile et al. (2002) para $T$. bournieri e $T$. sp. nr. mwanzai em ovos de $S$. cerealella e superiores aos observados por Zhang et al. (2001), para Trichogramma chilonis Ishii, T. nerudai, T. pretiosum e T. ostriniae criados em ovos de $P$. xylostella. Estes valores indicam que a razão finita de aumento pode variar de acordo com a espécie utilizada, e mostra que T. exiguum possui uma grande capacidade de adicionar indivíduos/ fêmea/dia nas diversas gerações avaliadas, sendo os melhores resultados encontrados no hospedeiro A. kuehniella.

\section{AGRADECIMENTOS}

Ao CNPq (Conselho Nacional de Desenvolvimento Científico e Tecnológico) pelo auxílio concedido. 


\section{REFERÊNCIAS BIBLIOGRÁFICAS}

BIRCH, L.C. 1948. The intrinsic rate of natural increase of an insect population. Journal of Animal Ecological 17: 15-26.

HANSEN, D.L.; BRODSGAAD, H.F.; ENKEGAARD. A. 1999. Life table characteristics of Macrolophus caliginosus preying upon Tetranychus urticae. Entomologia Experimentalis et Applicata 93: 269-275.

HAILE, A.T.; HASSAN, S.A.; OGOL, C.K.P.O.; BAUNGARTNER, J. 2002. Comparative life table analysis of Trichogramma bournieri Pintureal and Babalt and Trichogramma sp. Journal of Applied Entomology 127: 287-292.

NAVARRO, M.A. 1998. El Trichogramma spp.: Producción, Uso y Manejo en Colombia. Guadalajara de Buga, Impretec Ltda., 176p.

OLIVEIRA, H.N.; ZANUNCIO, J.C.; PRATISSOLI, D.; CRUZ. I. 2000. Parasitsm rate and viability of Trichogramma maxacalii (Hym.: Trichogrammatidae), parasitoid of the Eucalyptus defoliator Euselasia apisaon (Lep.: Riodinidae), on eggs of Anagasta huehniella (Lep.: Pyralidae). Forest Ecology and Management. Oxford, v.130, n.1/3, p.1-6.

PRATISSOLI, D.; PARRA, J.R.P. 2000. Desenvolvimento e exigências térmicas de Trichogramma pretiossum Riley, criados em duas traças do tomateiro. Pesquisa Agropecuária Brasileira 35: 12841-1288.
PRATISSOLI, D.; ZANUNCIO J.; RODRIGUES VIANNA U.; DE SOUZA ANDRADE J.; MAROTA GUIMARÃES E.; CURITIBA ESPINDULA M. 2004. Fertility life table of Trichogramma pretiosum and Trichogramma acacioi on eggs of Anagasta kuehniella at different temperatures. Pesquisa Agropecuária Brasileira 39: 193-196.

PARK, G.A.; OATMAN, E.R. 1982. Comparative life table, behavior and competition studies of Trichogramma brevicapillum and T. pretiosum. Entomologia Experimentalis et Applicata 32: 68-79.

SILVEIRA NETO, S.; NAKANO, O.; BARBIN. D.; VILA NOVA, N.A. 1976. Manual de ecologia dos insetos. São Paulo: Ceres, 419p.

SCHOLLER, M.; HASSAN, S. 2001. Comparative biology and life tables of Trichogramma evnescens and T. cacoeciae with Ephestia elutella as host at four constant temperatures. Entomologia Experimentalis et Applicata 98: 35-40.

ZANG, W. Q.; AGAMY, E.; HASSAN, S.A. 2001. Lifetable characteristics of four candidate species of the genus Trichogramma to control the diamondback moth Plutella xylostella (L.). Journal of Plant Diseases and Protection 108: 413-418.

ZUCCHI, R.A.; MONTEIRO, R.C. 1997. O gênero Trichogramma na América do Sul. In: PARRA, J.R.P.; ZUCCHI, R.A. Trichogramma e o controle biológico aplicado. Piracicaba: FEALQ, p.41-65. 